\title{
Optimal System of Subalgebras for the Reduction of the Navier-Stokes Equations
}

\author{
Sopita Khamrod ${ }^{1,2}$ \\ ${ }^{1}$ Department of Mathematics, Faculty of Science, Naresuan University, Phitsanulok, Thailand \\ ${ }^{2}$ Centre of Excellence in Mathematics, CHE, Bangkok, Thailand \\ Email:kuntimak@nu.ac.th
}

Received October 31, 2012; revised November 30, 2012; accepted December 7, 2012

\begin{abstract}
The purpose of this paper is to find the admitted Lie group of the reduction of the Navier-Stokes equations $\left(\left(U_{y y}(t, s, y)-U_{t}(t, s, y)\right) y-2 s U_{s y}(t, s, y)\right) y+\left(s^{2}+1\right) U_{s s}(t, s, y)+2 s U_{s}=0$ where $s=z / y \quad$ using the basic Lie symmetry method. This equation is constructed from the Navier-Stokes equations rising to a partially invariant solutions of the Navier-Stokes equations. Two-dimensional optimal system is determined for symmetry algebras obtained through classification of their subalgebras. Some invariant solutions are also found.
\end{abstract}

Keywords: Optimal System; Invariant Solutions; Partially Invariant Solutions; Navier-Stokes Equations

\section{Introduction}

Mathematical modeling is a basis for analyzing physical phenomena. Almost all fundamental equations of mathematical physics are nonlinear, and in general, are very difficult to solve explicitly. Group analysis is a method for constructing exact solutions of differential equations. This method uses the symmetry properties for constructing exact solutions. There are two types of solutions, the class of invariant solutions and partially invariant solutions which can be obtained by group analysis. Constructing of invariant and partially invariant solutions consists of some steps: choosing a subgroup of the admitted group, finding a representation of solution, substituting the representation into the studied system of equations and the study of compatibility of the obtained (reduced) system of equations.

This paper is devoted to use the basic Lie symmetry method for finding the admitted Lie group of the reduction of the Navier-Stokes equations,

$$
\begin{aligned}
& \left(\left(U_{y y}(t, s, y)-U_{t}(t, s, y)\right) y-2 s U_{s y}(t, s, y)\right) y \\
& +\left(s^{2}+1\right) U_{s s}(t, s, y)+2 s U_{s}=0
\end{aligned}
$$

where $U$ is a dependent variable and $t, s=z / y, y$ are independent variables. This equation is constructed from the Navier-Stokes equations. Subgroups for studying are taken from the part of optimal system of subalgebras considered for the gas dynamics equations [1]. One subgroup is not admitted the Navier-Stokes equations, par- tially invariant solutions can be found for the NavierStokes equations. These facts allow us to assume that one can construct partially invariant solution with respect to a Lie group, which is not necessary admitted. The proposed research will deal with two-dimensional optimal system of subalgebras for the reduction of the NavierStokes equations [1]. It is determined for symmetry algebras obtained through classification of their subalgebras. Example of some invariant solutions are also found. They can return to new solutions of the NavierStokes equations.

\section{Invariant and Partially Invariant Solutions}

The notion of invariant solution was introduced by Sophus Lie [2]. The notion of a partially invariant solution was introduced by Ovsiannikov [3]. This notion of partially invariant solutions generalizes the notion of an invariant solution, and extends the scope of applications of group analysis for constructing exact solutions of partial differential equations. The algorithm of finding invariant and partially invariant solutions consists of the following steps.

Let $L^{r}$ be a Lie algebra with the basis $X_{1}, \cdots, X_{r}$. The universal invariant $J$ consists of $s=m+n-r_{*}$ functionally independent invariants

$$
J=\left(J^{1}(x, u), J^{2}(x, u), \cdots, J^{m+n-r_{*}}(x, u)\right),
$$

where $n, m$ are the numbers of independent and depen- 
dent variables, respectively and $r_{*}$ is the total rank of the matrix composed by the coefficients of the generators $X_{i},(i=1,2, \cdots, r)$. If the rank of the Jacobi matrix $\frac{\partial\left(J^{1}, \cdots, J^{m+n-r_{*}}\right)}{\partial\left(u^{1}, \cdots, u^{m}\right)}$ is equal to $q$, then one can choose the first $q \leq m$ invariants $J^{1}, \cdots, J^{q}$ such that the rank of the Jacobi matrix $\frac{\partial\left(J^{1}, \cdots, J^{q}\right)}{\partial\left(u_{1}, \cdots, u_{m}\right)}$ is equal to $q$. A partially invariant solution is characterized by two integers: $\sigma \geq 0$ and $\delta \geq 0$. These solutions are also called $H(\sigma, \delta)$-solutions. The number $\sigma$ is called the rank of a partially invariant solution. This number gives the number of the independent variables in the representation of the partially invariant solution. The number $\delta$ is called the defect of a partially invariant solution. The defect is the number of the dependent functions which can not be found from the representation of partially invariant solution. The rank $\sigma$ and the defect $\delta$ must satisfy the conditions

$$
\begin{aligned}
& \sigma=\delta+n-r_{*} \geq 0, \delta \geq 0, \rho \leq \sigma<n, \\
& \max \left\{r_{*}-n, m-q, 0\right\} \leq \delta \leq \min \left\{r_{*}-1, m-1\right\},
\end{aligned}
$$

where $\rho$ is the maximum number of invariants which depends on the independent variables only. Note that for invariant solutions, $\delta=0$ and $q=m$.

For constructing a representation of a $H(\sigma, \delta)$ solution one needs to choose $l=m-\delta$ invariants and separate the universal invariant in two parts:

$$
\bar{J}=\left(J^{1}, \cdots, J^{l}\right), \overline{\bar{J}}=\left(J^{l+1}, J^{l+2}, \cdots, J^{m+n-r_{*}}\right) .
$$

The number $l$ satisfies the inequality $1 \leq l \leq q \leq m$. The representation of the $H(\sigma, \delta)$-solution is obtained by assuming that the first $l$ coordinates $\bar{J}$ of the universal invariant are functions of the invariants $\overline{\bar{J}}$ :

$$
\bar{J}=W(\overline{\bar{J}}) \text {. }
$$

Equation (2) form the invariant part of the representation of a solution. The next assumption about a partially invariant solution is that Equation (2) can be solved for the first $l$ dependent functions, for example,

$$
u^{i}=\phi^{i}\left(u^{l+1}, u^{l+2}, \cdots, u^{m}, x\right),(i=1, \cdots, l) .
$$

It is important to note that the functions $W^{i}$, $(i=1, \cdots, l)$ are involved in the expressions for the functions $\phi^{i},(i=1, \cdots, l)$. The functions $u^{l+1}, u^{l+2}, \cdots, u^{m}$ are called superfluous. The rank and the defect of the $H(\sigma, \delta)$-solution are $\delta=m-l$ and $\sigma=m+n-r_{*}-l=\delta+n-r_{*}$, respectively.

Note that if $\delta=0$, the above algorithm is the algorithm for finding a representation of an invariant solution. If $\delta \neq 0$, then Equation (3) do not define all dependent functions. Since a partially invariant solution satisfies the restrictions (2), this algorithm cuts out some particular solutions from the set of all solutions.

After constructing the representation of an invariant or partially invariant solution (3), it has to be substituted into the original system of equations. The system of equations obtained for the functions $W$ and superfluous functions $u^{k},(k=l+1,2, \cdots, m)$ is called the reduced system. This system is overdetermined and requires an analysis of compatibility. Compatibility analysis for invariant solutions is easier than for partially invariant solutions. Another case of partially invariant solutions which is easier than the general case occurs when $\overline{\bar{J}}$ only depends on the independent variables

$$
\begin{aligned}
& J^{l+1}=J^{l+1}(x), J^{l+2}=J^{l+2}(x), \cdots, \\
& J^{m+n-r_{*}}=J^{m+n-r_{*}}(x) .
\end{aligned}
$$

In this case, a partially invariant solution is called regular, otherwise it is irregular. The number $\sigma-\rho$ is called the measure of irregularity.

The process of studying compatibility consists of reducing the overdetermined system of partial differential equations to an involutive system. During this process different subclasses of $H(\sigma, \delta)$ partially invariant solutions can be obtained. Some of these subclasses can be $H_{1}\left(\sigma_{1}, \delta_{1}\right)$-solutions with subalgebra $H_{1} \subset H$. In this case $\sigma_{1} \geq \sigma, \delta_{1} \leq \delta$. The study of compatibility of partially invariant solutions with the same rank $\sigma_{1}=\sigma$, but with smaller defect $\delta_{1}<\delta$ is simpler than the study of compatibility for $H(\sigma, \delta)$-solutions. In many applications, there is a reduction of a $H(\sigma, \delta)$-solution to a $H_{1}(\sigma, 0)$ solution. In this case the $H(\sigma, \delta)$-solution is called reducible to an invariant solution. The problem of reduction to an invariant solution is important since invariant solutions are usually studied first.

\section{The Unsteady Navier-Stokes Equations}

Unsteady motion of incompressible viscous fluid is governed by the Navier-Stokes equations

$$
\begin{aligned}
& \mathbf{u}_{t}+\mathbf{u} \cdot \nabla \mathbf{u}=-\nabla p+\Delta \mathbf{u}, \\
& \nabla \cdot \mathbf{u}=0,
\end{aligned}
$$

where $\mathbf{u}=\left(u_{1}, u_{2}, u_{3}\right)=(u, v, w)$ is the velocity field, $p$ is the fluid pressure, $\nabla$ is the gradient operator in the three-dimensional space $\mathbf{x}=\left(x_{1}, x_{2}, x_{3}\right)=(x, y, z)$ and $\Delta$ is the Laplacian. A group classification of the Navier-Stokes equations in the three-dimensional case ${ }^{1}$ was done in [5]. The Lie group admitted by the NavierStokes equations is infinite. Its Lie algebra can be presented in the form of the direct sum $L^{\infty} \oplus L^{5}$, where the infinite-dimensional ideal $L^{\infty}$ is generated by the ope-

$\overline{{ }^{1} \text { A classification of the two-dimensional Navier-Stokes equations was }}$ studied in [4]. 
rators $^{2}$

$$
\begin{aligned}
& X_{\phi_{i}}=\phi_{i}(t) \partial_{x_{i}}+\phi_{i}^{\prime}(t) \partial_{u_{i}}-\phi_{i}^{\prime \prime}(t) x_{i} \partial_{p}, \\
& X_{\psi}=\psi(t) \partial_{p}
\end{aligned}
$$

with arbitrary functions $\phi_{i}(t),(i=1,2,3)$ and $\psi(t)$. The subalgebra $L^{5}$ has the following basis:

$$
\begin{aligned}
& Y=2 t \partial_{t}+x_{i} \partial_{x_{i}}-u_{i} \partial_{u_{i}}-2 p \partial_{p}, Z_{0}=\partial_{t}, \\
& Z_{i k}=x_{i} \partial_{x_{k}}-x_{k} \partial_{x_{i}}+u_{i} \partial_{u_{k}}-u_{k} \partial u_{i},(i<k \leq 3) .
\end{aligned}
$$

The Galilean algebra $L^{10}$ is contained in $L^{\infty} \oplus L^{5}$. Several articles [7-13] are devoted to invariant solutions of the Navier-Stokes equations ${ }^{3}$. While partially invariant solutions of the Navier-Stokes equations have been less studied ${ }^{4}$, there has been substantial progress in studying such classes of solutions of inviscid gas dynamics equations [18-25].

\section{The Reduction of the Navier-Stokes Equations}

The reduction of the Navier-Stokes equations to partial differential equation in three independent variables is described. In this section analysis of compatibility of regular partially invariant solutions with defect 1 and rank 1 of the subalgebras

$\left\{\partial_{x}, t \partial_{x}+\partial_{u}, \partial_{t}, t \partial_{t}+x \partial_{x}+y \partial_{y}+z \partial_{z}\right\}$ is given. Note that the generator $t \partial_{t}+x \partial_{x}+y \partial_{y}+z \partial_{z}$ is not admitted by the Navier-Stokes equations. The groups are taken from the optimal system constructed for the gas dynamics equations [26].

The Navier-Stokes equations are used in the component form:

$$
\begin{gathered}
u_{t}+u u_{x}+v u_{y}+w u_{z}=-p_{x}+u_{x x}+u_{y y}+u_{z z}, \\
v_{t}+u v_{x}+v v_{y}+w v_{z}=-p_{y}+v_{x x}+v_{y y}+v_{z z}, \\
w_{t}+u w_{x}+v w_{y}+w w_{z}=-p_{x}+w_{x x}+w_{y y}+w_{z z}, \\
u_{x}+v_{y}+w_{z}=0 .
\end{gathered}
$$

The dependent variables $u, v, w$ and $p$ are functions of the space variables $x, y, z$ and time $t$.

Invariants of the Lie group corresponding to subalgebra generated by $\left\{\partial_{x}, t \partial_{x}+\partial_{u}, \partial_{t}, t \partial_{t}+x \partial_{x}+y \partial_{y}+z \partial_{z}\right\}$ are

$$
v, w, p, z / y
$$

The representation of the regular partially invariant solution is

\footnotetext{
${ }^{2}$ There is still no complete classification of the subalgebras of the Lie algebra $L^{\infty} \oplus L^{5}$. Classification of infinite-dimensional subalgebras of this algebra was studied in [6].

${ }^{3}$ Short reviews devoted to invariant solutions of the Navier-Stokes equations can be found in [14-16].

${ }^{4}$ Firstly the approach of partially invariant solutions to the NavierStokes equations was applied in [17].
}

$$
v=V(s), w=W(s), p=P(s),
$$

where $s=z / y$. For the function $u=u(t, x, y, z)$ there is no restrictions. Substituting the representation of partially invariant solution (9) into the Navier-Stokes Equations (5)-(8), we obtain

$$
\begin{gathered}
u_{t}+u u_{x}+V u_{y}+W u_{z}-\left(u_{x x}+u_{y y}+u_{z z}\right)=0, \\
\left((W-s V) V^{\prime}-s P^{\prime}\right) y-\left(\left(s^{2}+1\right) V^{\prime \prime}+2 s V^{\prime}\right)=0, \\
\left((W-s V) W^{\prime}+P^{\prime}\right) y-\left(\left(s^{2}+1\right) W^{\prime \prime}+2 s W^{\prime \prime}\right)=0, \\
y u_{x}-\left(s V^{\prime}+W^{\prime}\right)=0 .
\end{gathered}
$$

Since $V$ and $W$ only depend on $s$, Equations (11) and (12) can be split with respect to $y$ :

$$
\begin{aligned}
& (W-s V) V^{\prime}-s P^{\prime}=0,(W-s V) W^{\prime}+P^{\prime}=0, \\
& \left(s^{2}+1\right) V^{\prime \prime}+2 s V^{\prime}=0,\left(s^{2}+1\right) W^{\prime \prime}+2 s W^{\prime}=0 .
\end{aligned}
$$

Solving Equation (15), we have

$$
V=C_{1} \arctan (s)+C_{2}, W=C_{3} \arctan (s)+C_{4} .
$$

Multiplying the first equation by $s$ and combining it with the second equation of (14), we obtain

$$
(W-s V)\left(V^{\prime}+s W^{\prime}\right)=0 .
$$

Let $W-s V=0$, then $C_{1}=C_{2}=C_{3}=C_{4}=0$. This means that $V=0, W=0$ and hence $P=C_{5}$. Substituting $V$ and $W$ in Equation (13), we have $u_{x}=0$. It means that $u$ depend on $t, y, z$ or $u=U(t, s, y)$. Equation (10) becomes

$$
\left(\left(U_{y y}-U_{t}\right) y-2 s U_{s y}\right) y+\left(s^{2}+1\right) U_{s s}+2 s U_{s}=0 .
$$

Thus, there is a solution of the Navier-Stokes equations of the type

$$
u=U(t, s, y), v=0, w=0, p=C_{5},
$$

where the function $U(t, s, y)$ satisfies Equation (16).

If $V^{\prime}+s W^{\prime}=0$, then $V=C_{2}, W=C_{4}$. In this case $P=C_{5}$. Note that the Galilei transformation applied to $V$ and $W$, also change $s$. Substituting $V$ and $W$ in Equation (13), we have $u_{x}=0$ or $u=U(t, s, y)$. Equation (10) becomes

$$
\begin{aligned}
& \left(\left(U_{y y}-C_{2} U_{y}-U_{t}\right) y-2 s U_{s y}\right) y+\left(s^{2}+1\right) U_{s s} \\
& +\left(\left(C_{2} y+2\right) s-C_{4} y\right) U_{s}=0 .
\end{aligned}
$$

Thus, there is a solution of the Navier-Stokes equations of the type

$$
u=U(t, s, y), v=C_{2}, w=C_{4}, p=C_{5},
$$

where the function $U(t, s, y)$ satisfies Equation (17).

These solutions are partially invariant solution with 
respect to the group which are not admitted Lie algebra $\left\{\partial_{x}, t \partial_{x}+\partial_{u}, \partial_{t}, t \partial_{t}+x \partial_{x}+y \partial_{y}+z \partial_{z}\right\}$.

\section{Admitted Group of Equation (16)}

In this section, the Lie group admitted by Equation (16) is studied. It was obtained from the Navier-Stokes equations and gives rise to a partially invariant solutions of the Navier-Stokes equations

$$
\begin{aligned}
& \left(\left(U_{y y}(t, s, y)-U_{t}(t, s, y)\right) y-2 s U_{s y}(t, s, y)\right) y \\
& +\left(s^{2}+1\right) U_{s s}(t, s, y)+2 s U_{s}=0
\end{aligned}
$$

where the function $U$ depends on $t, s, y$ and $s=z / y$. form

Assume that the generator has a representation of the

$$
\begin{aligned}
X= & \xi^{t}(t, s, y, U) \partial_{t}+\xi^{s}(t, s, y, U) \partial_{s} \\
& +\xi^{y}(t, s, y, U) \partial_{y}+\zeta^{U}(t, s, y, U) \partial_{U} .
\end{aligned}
$$

The second prolongation of the operator $X$ is

$$
\begin{aligned}
X^{(2)} & =X+\zeta^{U_{t}}(t, s, y, U) \partial_{U_{t}}+\zeta^{U_{s}}(t, s, y, U) \partial_{U_{s}} \\
& +\zeta^{U_{y}}(t, s, y, U) \partial_{U_{y}}+\zeta^{U_{t t}}(t, s, y, U) \partial_{U_{t t}} \\
& +\zeta^{U_{t s}}(t, s, y, U) \partial_{U_{t s}}+\zeta^{U_{t y}}(t, s, y, U) \partial_{U_{t y}} \\
& +\zeta^{U_{s y}}(t, s, y, U) \partial_{U_{s y}}+\zeta^{U_{s s}}(t, s, y, U) \partial_{U_{s s}} \\
& +\zeta^{U_{y y}}(t, s, y, U) \partial_{U_{y y}} .
\end{aligned}
$$

The coefficients of the prolonged operator are defined by formulae

$$
\begin{gathered}
\zeta^{U_{i}}=D_{i}\left(\zeta^{U}\right)-U_{j} D_{i}\left(\xi^{x_{j}}\right) ; \quad i, j=1,2,3 \\
\zeta^{U_{i_{1}, i_{2}}}=D_{i_{2}}\left(\zeta^{U_{i_{1}}}\right)-U_{i_{1}, j} D_{i_{2}}\left(\xi^{x_{j}}\right) ; \quad i, j=1,2,3 \\
D_{i}=\frac{\partial}{\partial x_{i}}+U_{i} \frac{\partial}{\partial U}+U_{i j} \frac{\partial}{\partial U_{j}}+\cdots ; \quad i, j=1,2,3 .
\end{gathered}
$$

Here we used the notations $x_{1}=t, x_{2}=s, x_{3}=y$ and for the derivatives

$$
U_{i}=D_{i}(U), U_{i j}=D_{j}\left(U_{i}\right) .
$$

The determining equations are

$$
\left.X^{(2)} F\right|_{[F=0]}=0 .
$$

All necessary calculations here were carried out on a computer using the symbolic manipulation program REDUCE.

The result of the calculations is the admitted Lie group

\begin{tabular}{|c|c|c|c|c|c|c|c|c|c|}
\hline$x$ & $X_{1}$ & $X_{2}$ & $X_{3}$ & $X_{4}$ & $X_{5}$ & $X_{6}$ & $X_{7}$ & $X_{8}$ & $X_{9}$ \\
\hline$X_{1}$ & 0 & 0 & 0 & $2 X_{1}$ & $2 X_{2}$ & 0 & $2 X_{2}$ & $4 X_{4}$ & 0 \\
\hline$X_{2}$ & 0 & 0 & 0 & $X_{2}$ & $-X_{9}$ & $X_{3}$ & 0 & $2 X_{5}$ & 0 \\
\hline$X_{3}$ & 0 & 0 & 0 & $X_{3}$ & 0 & $-X_{2}$ & $-X_{9}$ & $2 X_{7}$ & 0 \\
\hline$X_{4}$ & $-2 X_{1}$ & $-X_{2}$ & $-X_{3}$ & 0 & $-X_{5}$ & 0 & $X_{7}$ & $2 X_{8}$ & 0 \\
\hline$X_{5}$ & $-2 X_{2}$ & $X_{9}$ & 0 & $-X_{5}$ & 0 & $X_{7}$ & 0 & 0 & 0 \\
\hline$X_{6}$ & 0 & $-X_{3}$ & $X_{2}$ & 0 & $-X_{7}$ & 0 & $X_{5}$ & 0 & 0 \\
\hline$X_{7}$ & $-2 X_{3}$ & 0 & $X_{9}$ & $-X_{7}$ & 0 & $-X_{5}$ & 0 & 0 & 0 \\
\hline$X_{8}$ & $-4 X_{4}$ & $-2 X_{5}$ & $-2 X_{7}$ & $-2 X_{8}$ & 0 & 0 & 0 & 0 & 0 \\
\hline$X_{9}$ & 0 & 0 & 0 & 0 & 0 & 0 & 0 & 0 & 0 \\
\hline
\end{tabular}
with the basis of the generators:

$$
\begin{aligned}
& X_{1}=\partial_{t}, X_{2}=\frac{1}{y} \partial_{s}, X_{3}=\frac{s}{y} \partial_{s}-\partial_{y}, \\
& X_{4}=2 t \partial_{t}+y \partial_{y}-U \partial_{U}, \\
& X_{5}=\frac{2 t}{y} \partial_{s}-s y U \partial_{U}, X_{6}=\left(s^{2}+1\right) \partial_{s}-s y \partial_{y}, \\
& X_{7}=\frac{2 t s}{y} \partial_{s}-2 t \partial_{y}+y U \partial_{U}, \\
& X_{8}=4 t^{2} \partial_{t}+4 t y \partial_{y}-\left(4 t+\left(s^{2}+1\right) y^{2}\right) U \partial_{U}, \\
& X_{9}=U \partial_{U}, X_{10}=b(t, s, y) \partial_{U},
\end{aligned}
$$

where $b(t, s, y)$ is an arbitrary solution of

$$
\left(\left(b_{y y}-b_{t}\right) y-2 s b_{s y}\right) y+\left(s^{2}+1\right) b_{s s}+2 s b_{s}=0 .
$$

\section{Optimal System of Subalgebras}

The problem is to construct subalgebras of the algebra $L^{10}$, which can be a source of invariant solutions of Equation (1). The classification of subalgebras can be done relatively easy for small dimensions. The optimal system of subalgebras of the Lie algebra spanned by the generators $X_{1}, \cdots, X_{9}$ are constructed here.

The table of commutators $\left[X_{i}, X_{j}\right]$ is

Inner automorphisms [24] are constructed with the help of the table of commutators.

To construct inner automorphisms, one has to solve the Lie equations. For example, for the automorphism $A_{1}$, one has the system of ordinary differential equations

$$
\frac{\mathrm{d} \bar{x}_{1}}{\mathrm{~d} a}=-2 \bar{x}_{4}, \quad \frac{\mathrm{d} \bar{x}_{2}}{\mathrm{~d} a}=-2 \bar{x}_{5}, \quad \frac{\mathrm{d} \bar{x}_{3}}{\mathrm{~d} a}=-2 \bar{x}_{7}, \frac{\mathrm{d} \bar{x}_{4}}{\mathrm{~d} a}=-4 \bar{x}_{8} .
$$

and the initial values at $a=0$

$$
\bar{x}_{1}=x_{1}, \bar{x}_{2}=x_{2}, \bar{x}_{3}=x_{3}, \bar{x}_{4}=x_{4} .
$$

Therefore, the automorphism $A_{1}$ only changes the coordinates $x_{1}, x_{2}, x_{3}$ and $x_{4}$ by the formulae 


$$
\begin{aligned}
& \bar{x}_{1}=x_{1}-2 a_{1} x_{4}+4 a_{1}^{2} x_{8}, \bar{x}_{2}=x_{2}-2 a_{1} x_{5}, \\
& \bar{x}_{3}=x_{3}-2 a_{1} x_{7}, \bar{x}_{4}=x_{4}-4 a_{1} x_{8} .
\end{aligned}
$$

The remaining coordinates are unchanged.

In the same way, one obtains the automorphisms $A_{i}(i=2, \cdots, 9)$ :

$$
\begin{aligned}
& A_{2}: \bar{x}_{2}=x_{2}-a_{2} x_{4}, \bar{x}_{3}=x_{3}-a_{2} x_{6}, \\
& \bar{x}_{5}=x_{5}-2 a_{2} x_{8}, \bar{x}_{9}=x_{9}+a_{2} x_{5}-a_{2}^{2} x_{8} \\
& A_{3}: \bar{x}_{2}=x_{2}+a_{3} x_{6}, \bar{x}_{3}=x_{3}-a_{3} x_{4} \text {, } \\
& \bar{x}_{7}=x_{7}-2 a_{2} x_{8}, \bar{x}_{9}=x_{9}+a_{3} x_{7}-a_{3}^{2} x_{8} \\
& A_{4}: \bar{x}_{1}=x_{1} \mathrm{e}^{2 a_{4}}, \bar{x}_{2}=x_{2} \mathrm{e}^{2 a_{4}}, \bar{x}_{3}=x_{3} \mathrm{e}^{2 a_{4}}, \\
& \bar{x}_{5}=x_{5} \mathrm{e}^{-2 a_{4}}, \bar{x}_{7}=x_{7} \mathrm{e}^{-2 a_{4}}, \bar{x}_{8}=x_{8} \mathrm{e}^{-2 a_{4}} \\
& A_{5}: \quad \bar{x}_{2}=x_{2}+2 a_{5} x_{1}, \bar{x}_{5}=x_{5}+a_{5} x_{4}, \\
& \bar{x}_{7}=x_{7}-a_{5} x_{6}, \bar{x}_{9}=x_{9}-a_{5} x_{2}-a_{5}^{2} x_{1} \\
& A_{6}: \bar{x}_{2}=x_{2} \cos \left(a_{6}\right)-x_{3} \sin \left(a_{6}\right) \text {, } \\
& \bar{x}_{3}=x_{2} \sin \left(a_{6}\right)+x_{3} \cos \left(a_{6}\right) \text {, } \\
& \bar{x}_{5}=x_{5} \cos \left(a_{6}\right)-x_{7} \sin \left(a_{6}\right) \text {, } \\
& \bar{x}_{7}=x_{5} \sin \left(a_{6}\right)+x_{7} \cos \left(a_{6}\right) \\
& A_{7}: \quad \bar{x}_{3}=x_{3}+2 a_{7} x_{1}, \bar{x}_{5}=x_{5}+a_{7} x_{6}, \\
& \bar{x}_{7}=x_{7}+a_{7} x_{4}, \bar{x}_{9}=x_{9}-a_{7} x_{3}-a_{7}^{2} x_{1} \\
& A_{8}: \bar{x}_{4}=x_{4}+4 a_{8} x_{1}, \bar{x}_{5}=x_{5}+2 a_{8} x_{2} \text {, } \\
& \bar{x}_{7}=x_{7}+2 a_{8} x_{3}, \bar{x}_{8}=x_{8}+2 a_{8} x_{4}+4 a_{8}^{2} x_{1} \\
& A_{9}: \quad \bar{x}_{1}=x_{1}, \bar{x}_{2}=x_{2}, \bar{x}_{3}=x_{3} \text {, } \\
& \bar{x}_{4}=x_{4}, \bar{x}_{5}=x_{5}, \bar{x}_{6}=x_{6} \text {, } \\
& \bar{x}_{7}=x_{7}, \bar{x}_{8}=x_{8}, \bar{x}_{9}=x_{9} .
\end{aligned}
$$

Also there is the involution

$$
E: \quad \bar{x}_{3}=-x_{3}, \quad \bar{x}_{6}=-x_{6}, \quad \bar{x}_{7}=-x_{7} .
$$

\subsection{Decomposition of the Algebra $L^{9}$}

Before constructing an optimal system, let us study the algebraic structure of the algebra $L^{9}$. The algebra $L^{9}$ is decomposed as $I \oplus L^{4}$, where $I=\left\{X_{2}, X_{3}, X_{5}, X_{7}, X_{9}\right\}$ is an ideal and $L^{4}=\left\{X_{1}, X_{4}, X_{6}, X_{8}\right\}$ is a subalgebra. According to the algorithm for constructing an optimal system of the algebra $L^{9}$, we use the two-step algorithm developed in [21]. First, an optimal system of subalgebras of the algebra $L^{4}$ is obtained. The next step is to glue the subalgebras from the optimal system of subalgebras of the algebra $L^{4}$ and the ideal $I$ together.

Any subalgebra of a Lie algebra is completely defined by its basis generators. Any vector of the basis is a linear combination of the basis of generator of this Lie algebra. Hence, the subalgebra is completely defined by co- efficients of these linear combinations. For example, let $L^{k}=\left\{Y_{1}, Y_{2}, \cdots, Y_{k}\right\}$ be a $k$-dimensional subalgebra of the algebra $L^{9}$. Operators $Y_{i},(i=1,2, \cdots, k)$ are

$$
Y_{i}=\sum_{\alpha=1}^{9} X_{i \alpha} X_{\alpha}, i=1, \cdots, k \text {. }
$$

Conditions for $L^{k}$ to be a subalgebra are

$$
\left[Y_{i}, Y_{j}\right]=\sum_{\alpha=1}^{k} C_{i j}^{\alpha} Y_{\alpha} ; i, j=1,2, \cdots, k .
$$

For a classification of subalgebra, the coefficients $C_{i j}^{\alpha}$ have to be simplified by using the automorphism and subalgebra conditions.

\subsection{Classification of the Algebra $L^{4}$}

Let us classify the algebra $L^{4}=\left\{X_{1}, X_{4}, X_{6}, X_{8}\right\}$. The table of commutators of the algebra $L^{4}$ is

\begin{tabular}{c|cccc}
\hline$X_{i}$ & $X_{j}$ & $X_{4}$ & $X_{6}$ & $X_{8}$ \\
\hline$X_{1}$ & 0 & $2 X_{1}$ & 0 & $4 X_{4}$ \\
$X_{4}$ & $-2 X_{1}$ & 0 & 0 & $2 X_{8}$ \\
$X_{6}$ & 0 & 0 & 0 & 0 \\
$X_{8}$ & $-4 X_{4}$ & $-2 X_{8}$ & 0 & 0 \\
\hline
\end{tabular}

Since the generator $X_{6}$ composes the center, the optimal system of subalgebras of $L^{4}=\left\{X_{1}, X_{4}, X_{6}, X_{8}\right\}$ can be easily constructed by classifying the subalgebra $L^{3}=\left\{X_{1}, X_{4}, X_{8}\right\}$ and gluing it with the center $\left\{X_{6}\right\}$. The idea of construction is as follows.

Let a subalgebra $L^{r}$ of dimension $r \leq 4$ be formed by the operators

$$
Y_{i}=a_{i 1} X_{1}+a_{i 2} X_{4}+a_{i 3} X_{6}+a_{i 4} X_{8}, i=1, \cdots, r
$$

where $a_{i j},(i=1, \cdots, r ; j=1,2,3,4)$ are arbitrary constants.

For the classification of $L^{4}$ we need to study two steps.

1) All coefficients $a_{i 3}$ are zero, $a_{i 3}=0(i=1,2,3,4)$, it means that we will construct an optimal system of the subalgebra $L^{3}=\left\{X_{1}, X_{4}, X_{8}\right\}$.

2) At least one of the coefficients of $a_{i 3}$ is not equal to zero.

Let us study the first step, and construct an optimal system of the subalgebra $L^{3}$. For convenience, we will denote the generators $X_{i}$ by $i$.

\subsubsection{One-Dimensional Subalgebras of the Algebra} $L^{3}$

Let $Y=x_{1} \mathbf{1}+x_{4} \mathbf{4}+x_{8} \mathbf{8}$ which forms a one-dimensional subalgebra of the algebra $L^{3}$. The process of simplification of the coefficients of the operator $Y$ is separated into the following cases. 
Case 1. Assume that $x_{8} \neq 0$. Then one can divide $Y$ by $X_{8}$. Hence, without loss of generality one can consider

$$
Y=x_{1} \mathbf{1}+x_{4} \mathbf{4}+x_{8} \mathbf{8}
$$

By means of transformation $A_{1}$, it can transformed to an operator with $x_{4}=0$.

Case 1.1. Let $x_{1} \neq 0$. By means of transformation $A_{4}$, one can transform it to $\varepsilon \mathbf{1}+\mathbf{8}$, where $\varepsilon= \pm 1$.

Case 1.2. Let $x_{1}=0$, then the representative of the class is the operator $\mathbf{8}$.

Case 2. Assume that $x_{8}=0$. Then one has $Y=x_{1} 1+x_{4} 4$.

Case 2.1. Let $x_{4} \neq 0$. Dividing the operator $Y$ by $x_{4}$, one obtains $Y=x_{1} 1+x_{4} 4$. By using the automorphism $A_{1}$, the operator $Y$ is transformed to $X_{4}$.

Case 2.2. Let $x_{4}=0$, then $Y=1$.

\subsubsection{Two-Dimensional Subalgebras of the Algebra $\boldsymbol{L}^{3}$}

Let a subalgebra be formed by the operators

$$
Y_{i}=a_{i 1} \mathbf{1}+a_{i 2} \mathbf{4}+a_{i 3} \mathbf{8}, i=1,2
$$

where $a_{i j},(i=1,2 ; j=1,2,3)$ are arbitrary constants. Note that the rank of the matrix $\left(\begin{array}{lll}a_{11} & a_{12} & a_{13} \\ a_{21} & a_{22} & a_{23}\end{array}\right)$ is equal to two.

Case 1. Assume that $a_{13} \neq 0$. We can divide $Y_{1}$ by $a_{13}$. Hence, by subtracting the operator $\left(a_{23} / a_{13}\right) Y_{1}$ from $Y_{2}$, one can assume $a_{23}=0$ and $a_{21}^{2}+a_{22}^{2} \neq 0$. Using the automorphisms $A_{1}$, the operator $Y_{1}$ is transformed to $Y_{1}=a_{11} \mathbf{1}+\mathbf{8}$. The subalgebra condition gives

$$
\left[a_{11} \mathbf{1}+\mathbf{8}, a_{21} \mathbf{1}+a_{22} \mathbf{8}\right]=\alpha\left(a_{11} \mathbf{1}+\mathbf{8}\right)+\beta\left(a_{21} \mathbf{1}+a_{22} \mathbf{8}\right)
$$

where $\alpha$ and $\beta$ are arbitrary constants. Calculating the left hand side and comparing the coefficients on the left hand side with coefficients on the right hand side, one has

$$
2 a_{11} a_{22} \mathbf{1}-4 a_{21} \mathbf{4}-2 a_{22} \mathbf{8}=\left(\alpha a_{11}+\beta a_{21}\right) \mathbf{1}+\beta a_{22} \mathbf{4}+\alpha \mathbf{8} .
$$

Therefore

$$
2 a_{11} a_{22}=\alpha a_{11}+\beta a_{21},-4 a_{21}=\beta a_{22},-2 a_{22}=\alpha .
$$

Further consideration depends on values of the coefficients $a_{11}, a_{21}, a_{22}$. If $a_{22}=0$, then $a_{21}=0$ which is a contradiction to the condition $a_{21}^{2}+a_{22}^{2} \neq 0$. Hence, $a_{22} \neq 0$. One can assume that $a_{22}=1$. Therefore $\alpha=-2, \beta=-4 a_{11}$, and $a_{11}=-a_{21}^{2}$.

Case 1.1. If $a_{21} \neq 0$, then using the automorphism $A_{4}$, the operators $Y_{1}$ and $Y_{2}$ are transformed to $Y_{1}=\mathbf{8}-\mathbf{1}, Y_{2}=\mathbf{4}+\mathbf{1}$.

Case 1.2. If $a_{21}=0$, then the operators $Y_{1}$ and $Y_{2}$ are $Y_{1}=\mathbf{8}, Y_{2}=\mathbf{4}$.
Case 2. Assume that $a_{13}=0$. If $a_{23} \neq 0$, then by exchanging $Y_{1}$ and $Y_{2}$, this becomes the previous case. Hence, one can take $a_{23}=0$. Therefore, the operators are $Y_{1}=a_{11} 1+a_{12} 4, Y_{2}=a_{21} 1+a_{22} 4$. Because the rank of the matrix

$$
\left(\begin{array}{ll}
a_{11} & a_{12} \\
a_{21} & a_{22}
\end{array}\right)
$$

is equal to 2 , then by taking linear combinations of the operators $Y_{1}$ and $Y_{2}$ they can be transformed to $Y_{1}=\mathbf{1}$ and $Y_{2}=\mathbf{4}$.

\subsubsection{Three-Dimensional Subalgebras of the Algebra $\boldsymbol{L}^{3}$}

Let a subalgebra be formed by these operators

$$
Y_{i}=a_{i 1} \mathbf{1}+a_{i 2} \mathbf{4}+a_{i 3} \mathbf{8}, i=1,2,3
$$

where $a_{i j},(i=1,2,3 ; j=1,2,3)$ are arbitrary constants. Since the rank of the matrix

$$
\left(\begin{array}{lll}
a_{11} & a_{12} & a_{13} \\
a_{21} & a_{22} & a_{23} \\
a_{31} & a_{32} & a_{33}
\end{array}\right)
$$

is equal to three, the basis if this subalgebra can be taken as

$$
Y_{1}=\mathbf{1}, Y_{2}=\mathbf{4}, Y_{3}=\mathbf{8}
$$

\subsubsection{Optimal System of Subalgebras of the Algebra} $L^{3}=\{1,4,8\}$

The result of classifying the algebra $\boldsymbol{L}^{3}=\{\mathbf{1}, \mathbf{4 , 8}\}$ is the following:

\begin{tabular}{ccc}
\hline \multicolumn{3}{c}{ Dimension } \\
\hline 1 & 2 & 3 \\
1 & 1,4 & $\mathbf{1 , 4 , 8}$ \\
4 & 4,8 & \\
8 & $1-8,1+4$ & \\
$\varepsilon 1+8$ & & \\
\hline
\end{tabular}

where $\varepsilon= \pm 1$.

\subsection{Optimal System of Subalgebras of the Algebra $L^{4}=\{1,4,6,8\}$}

Let us consider the second step where at least one of the coefficients $a_{i 3}$ is not equal to zero. Without loss of generality one can assume that

$$
\begin{aligned}
& Y_{1}=\mathbf{6}+a_{11} \mathbf{1}+a_{12} \mathbf{4}+a_{14} \mathbf{8} \\
& Y_{i}=a_{i 1} \mathbf{1}+a_{i 2} \mathbf{4}+a_{i 4} \mathbf{8}, i=2, \cdots, r, r \leq 4 .
\end{aligned}
$$

Using the conditions for $L^{4}$ to be a subalgebra, one 
obtains

$$
\left[Y_{i}, Y_{j}\right]=\alpha_{i j} \mathbf{6}+\beta_{i j} \mathbf{1}+\gamma_{i j} \mathbf{4}+\sigma_{i j} \mathbf{8} ; \quad i, j=1,2, \cdots, 4 .
$$

Because $L^{3}=\{\mathbf{1 , 4 , 8}\}$ is a subalgebra and the generator 6 forms the center, then

$$
\left[Y_{i}, Y_{j}\right]=\hat{\beta}_{i j} \mathbf{1}+\hat{\gamma}_{i j} \mathbf{4}+\hat{\sigma}_{i j} \mathbf{8} ; \quad i, j=1,2, \cdots, 4 .
$$

Comparing the coefficients, one obtains $\alpha_{i j}=0 ;, j=1,2, \cdots, 4$. Because of these results and since the algebra $L^{3}=\{\mathbf{1}, \mathbf{4}, \mathbf{8}\}$ has already been classified, therefore this allows simplifying the process of constructing the optimal system of the algebra $L^{4}$. This process construct by using the result of the optimal system of algebra $L^{3}$ : we have to classify each optimal system of subalgebras of $L^{3}$ together with the generator $Y_{1}=\mathbf{6}+a_{11} \mathbf{1}+a_{12} \mathbf{4}+a_{14} \mathbf{8}$. Here we give one example of this process. Other elements of the optimal system of the algebra $L^{4}$ are constructed in the similar way.

Let us consider the subalgebra $\{\mathbf{1}-\mathbf{8}, \mathbf{1}+\mathbf{4}\}$. For constructing three-dimensional subalgebras of the algebra $L_{4}$ one considers

$$
Y_{1}=\mathbf{6}+a_{11} \mathbf{1}+a_{12} \mathbf{4}+a_{14} \mathbf{8}, Y_{2}=\mathbf{1}-\mathbf{8}, Y_{3}=\mathbf{1}+\mathbf{4} .
$$

Since $Y_{1}$ can be written as:

$$
Y_{1}=\mathbf{6}+\left(a_{11}-a_{12}+a_{14}\right) \mathbf{1}+a_{12}(\mathbf{1}+\mathbf{4})+a_{14}(\mathbf{8}-\mathbf{1}),
$$

by forming a linear combination with $Y_{2}$ and $Y_{3}$, the operator $Y_{1}$ can be taken in the form $Y_{1}=\mathbf{6}+\bar{a}_{11} \mathbf{1}$. The subalgebra conditions gives

$$
\begin{aligned}
& {\left[\mathbf{6}+\bar{a}_{11} \mathbf{1}, \mathbf{1}-\mathbf{8}\right]=-4 \bar{a}_{11} \mathbf{4}} \\
& =\alpha\left(\mathbf{6}+\bar{a}_{11} \mathbf{1}\right)+\beta(\mathbf{1}-\mathbf{8})+\gamma(\mathbf{1}+\mathbf{4})
\end{aligned}
$$

where $\alpha, \beta$ and $\gamma$ are arbitrary constants. Comparing the coefficients on the left side with the coefficients on the right side, one obtains

$$
\alpha=0, \beta=0, \gamma=0, \bar{a}_{11}=0 .
$$

Thus, one obtains that $Y_{1}=\mathbf{6}$, and the subalgebra is $\{6,1-8,1+4\}$.

The result of calculation is an optimal system of subalgebras of the algebra $L^{4}=\{\mathbf{1 , 4 , 6 , 8}\}$ which is

\begin{tabular}{cccc}
\hline \multicolumn{4}{c}{ Dimension } \\
\hline 1 & 2 & 3 & 4 \\
\hline 1 & 1,4 & $1,4,6$ & $1,4,6,8$ \\
4 & 4,6 & $1,4,8$ & \\
6 & 4,8 & $4,6,8$ & \\
8 & $1,6+\beta 4$ & $6,1+4,1-8$ & \\
$1+6$ & $8,6+\beta 4$ & & \\
$\varepsilon 1+8$ & $\varepsilon 1+8,6$ & & \\
$4+6$ & $1+4,1-8$ & & \\
$8+6$ & & & \\
$\varepsilon 1+6+8$ & & & \\
\hline
\end{tabular}

where $\beta$ is an arbitrary real parameter and $\varepsilon= \pm 1$.

\subsection{Optimal System of Subalgebras of the Algebra $\boldsymbol{L}^{\mathbf{9}}$}

After constructing an optimal system of subalgebras of the algebra $L^{4}$, the next step is the construction of an optimal system of subalgebras of the algebra

$L^{9}=\{\mathbf{1}, \mathbf{2}, \mathbf{3}, \mathbf{4}, \mathbf{5}, \mathbf{6}, \mathbf{7}, \mathbf{8}, \mathbf{9}\}$, by gluing subalgebras from the optimal system of subalgebras of the algebra $L^{4}$ and the ideal $I=\{2,3,5,7,9\}$ together.

As it was seen for the algebra $L^{4}$, the process of constructing an optimal system of subalgebras of the algebra $L^{9}$ by gluing the algebra $L^{4}$ and the ideal $I$ consists of the following steps. In the first step, the vectors

$$
\begin{aligned}
& Y_{i}=\sum_{j=\{2,3,5,7,9\}} a_{i j} X_{j}+\sum_{j=\{1,4,6,8\}} b_{i j} X_{j},(i=1,2, \cdots, k), \\
& Y_{i+k}=\sum_{j=\{2,3,5,7,9\}} c_{i j} X_{j} \quad(i=1,2, \cdots, s),
\end{aligned}
$$

are composed. Here the vectors

$$
\sum_{j=\{1,4,6,8\}} b_{i j} X_{j}
$$

are basis elements from one of the $k$-dimensional subalgebras $L^{k}$ of the optimal system of the algebra $L^{4}$. In matrix form, this step can be explained by the construction of the matrix

\begin{tabular}{cc}
\hline 23579 & 1468 \\
\hline$A$ & $B$ \\
$C$ & 0 \\
\hline
\end{tabular}

where the matrices $\boldsymbol{A}, \boldsymbol{B}$ and $\boldsymbol{C}$ consist of the coefficients $a_{i j}, b_{i \alpha}, c_{\beta j}$,

$(i=1,2, \cdots, k ; j=2,3,5,7,9 ; \alpha=1,4,6,8 ; \beta=1,2, \cdots, s)$.

In this step, the matrix $\boldsymbol{A}$ is arbitrary. The rank of the matrix

$$
\left(\begin{array}{ll}
\boldsymbol{A} & \boldsymbol{B} \\
\boldsymbol{C} & \mathbf{0}
\end{array}\right)
$$

is equal to $k+s$ and this is the dimension of the subalgebra of the algebra $L^{9}$. The matrix $\boldsymbol{C}$ is chosen to be the simplest by taking linear combinations of it columns and has to take all possible values of the given rank $s$. Note also that the matrix $\boldsymbol{A}$ can be simplified with the help of the matrix $\boldsymbol{C}$.

The next step is the process of checking the subalgebra conditions and checking linear dependence of commutators on the basis generators of the subalgebra.

In this manuscript, we study only two-dimensional subalgebras of the algebra $L^{9}$, because the two-dimen- 
sional subalgebras allow obtaining invariant solutions which reduce the initial system of partial differential equations to a system of ordinary differential equations.

Let us give an example for constructing two-dimensional subalgebras, using the subalgebra $\{\varepsilon \mathbf{1}+\mathbf{8}\}$. The maximum possible dimension of a subalgebra of the algebra $L^{4}$ after gluing a subalgebra to the ideal $I$ is two. In this case, the matrix $C$ is a $1 \times 5$ matrix, the rank of which is equal to one:

\begin{tabular}{ccccccccc}
\hline $\mathbf{2}$ & $\mathbf{3}$ & $\mathbf{5}$ & $\mathbf{7}$ & $\mathbf{9}$ & $\mathbf{1}$ & $\mathbf{4}$ & $\mathbf{6}$ & $\mathbf{8}$ \\
\hline$a_{12}$ & $a_{13}$ & $a_{15}$ & $a_{17}$ & $a_{19}$ & $\varepsilon$ & 0 & 0 & 1 \\
$c_{22}$ & $c_{23}$ & $c_{25}$ & $c_{27}$ & $c_{29}$ & 0 & 0 & 0 & 0 \\
\hline
\end{tabular}

By virtue of the automorphism $A_{6}$ :

$$
\begin{aligned}
& \bar{x}_{2}=x_{2} \cos \left(a_{6}\right)-x_{3} \sin \left(a_{6}\right), \\
& \bar{x}_{3}=x_{2} \sin \left(a_{6}\right)+x_{3} \cos \left(a_{6}\right), \\
& \bar{x}_{5}=x_{5} \cos \left(a_{6}\right)-x_{7} \sin \left(a_{6}\right), \\
& \bar{x}_{7}=x_{5} \sin \left(a_{6}\right)+x_{7} \cos \left(a_{6}\right) .
\end{aligned}
$$

We can consider three cases:

1) $c_{22}^{2}+c_{23}^{2} \neq 0$,

2) $c_{22}^{2}+c_{23}^{2}=0, c_{25}^{2}+c_{27}^{2} \neq 0$,

3) $c_{22}^{2}+c_{23}^{2}=0, c_{25}^{2}+c_{27}^{2}=0, c_{29} \neq 0$.

Case 1. By using the automorphism $A_{6}$ one can assume $c_{22}=1, c_{23}=0$. In this case, by means of linear combinations and by the automorphisms $A_{2}, A_{3}, A_{5}, A_{7}$ the table of coefficients is transformed to

\begin{tabular}{ccccccccc}
\hline $\mathbf{2}$ & $\mathbf{3}$ & $\mathbf{5}$ & $\mathbf{7}$ & $\mathbf{9}$ & $\mathbf{1}$ & $\mathbf{4}$ & $\mathbf{6}$ & $\mathbf{8}$ \\
\hline 0 & 0 & 0 & 0 & $a_{19}$ & $\varepsilon$ & 0 & 0 & 1 \\
1 & 0 & $c_{25}$ & $c_{27}$ & $c_{29}$ & 0 & 0 & 0 & 0 \\
\hline
\end{tabular}

The subalgebra conditions give

$$
\begin{aligned}
& {\left[\varepsilon \mathbf{1}+\mathbf{8}+a_{19} \mathbf{9}, \mathbf{2}+c_{25} \mathbf{5}+c_{27} \mathbf{7}+c_{29} \mathbf{9}\right]} \\
& =2 \varepsilon c_{25} \mathbf{2}+2 \varepsilon c_{27} \mathbf{3}-25 \\
& =\alpha\left(\varepsilon \mathbf{1}+\mathbf{8}+a_{19} \mathbf{9}\right)+\beta\left(\mathbf{2}+c_{25} \mathbf{5}+c_{27} \mathbf{7}+c_{29} \mathbf{9}\right),
\end{aligned}
$$

where the coefficients $\alpha$ and $\beta$ are arbitrary constants. Comparing the coefficients, one obtains

$$
\alpha=0, \beta= \pm 2, \varepsilon=-1, c_{27}=0, c_{29}=0, c_{25}= \pm 1 .
$$

Therefore, in this case the subalgebra is $\left\{-\mathbf{1}+\mathbf{8}+a_{19} \mathbf{9}, \mathbf{2}+\varepsilon \mathbf{5}\right\}$.

Case 2. Since $c_{22}^{2}+c_{23}^{2}=0$, or $c_{22}=0, c_{23}=0$. Because of $c_{25}^{2}+c_{27}^{2} \neq 0$, by virtue of the automorphism $A_{6}$ one can take $c_{25}=1, c_{27}=0$. By means of linear combinations and by the automorphisms $A_{2}, A_{3}, A_{5}, A_{7}$, the coefficients are transformed to

\begin{tabular}{ccccccccc}
\hline $\mathbf{2}$ & $\mathbf{3}$ & $\mathbf{5}$ & $\mathbf{7}$ & $\mathbf{9}$ & $\mathbf{1}$ & $\mathbf{4}$ & $\mathbf{6}$ & $\mathbf{8}$ \\
\hline 0 & 0 & 0 & 0 & $a_{19}$ & $\varepsilon$ & 0 & 0 & 1 \\
0 & 0 & 1 & 0 & $c_{29}$ & 0 & 0 & 0 & 0 \\
\hline
\end{tabular}

The subalgebra condition gives

$$
\begin{aligned}
& {\left[\varepsilon \mathbf{1}+\mathbf{8}+a_{19} \mathbf{9}, \mathbf{5}+c_{29} \mathbf{9}\right]=2 \varepsilon \mathbf{2}} \\
& =\alpha\left(\varepsilon \mathbf{1}+\mathbf{8}+a_{19} \mathbf{9}\right)+\beta\left(\mathbf{5}+c_{29} \mathbf{9}\right),
\end{aligned}
$$

where the coefficients $\alpha$ and $\beta$ are arbitrary constants. Comparing the coefficients, one obtains

$$
\alpha=0, \beta=0, \varepsilon=0 \text {. }
$$

This is a contradiction to $\varepsilon \neq 0$. Therefore, there exists no subalgebra in this case.

Case 3. Assume that $c_{22}^{2}+c_{23}^{2}=0, c_{25}^{2}+c_{27}^{2}=0$ and $c_{29} \neq 0$, or $c_{22}=0, c_{23}=0, c_{25}=0, c_{27}=0$. Since $c_{29} \neq 0$, without loss of generality one can choose $c_{29}=1$. By taking linear combinations and by virtue of the automorphism $A_{2}, A_{3}, A_{5}, A_{7}$ the table of coefficients can be transformed to

\begin{tabular}{lllllllll}
\hline $\mathbf{2}$ & $\mathbf{3}$ & $\mathbf{5}$ & $\mathbf{7}$ & $\mathbf{9}$ & $\mathbf{1}$ & $\mathbf{4}$ & $\mathbf{6}$ & $\mathbf{8}$ \\
\hline 0 & 0 & 0 & 0 & 0 & $\varepsilon$ & 0 & 0 & 1 \\
0 & 0 & 0 & 0 & 1 & 0 & 0 & 0 & 0 \\
\hline
\end{tabular}

The subalgebra conditions give

$$
[\varepsilon \mathbf{1}+\mathbf{8}, \mathbf{9}]=0=\alpha(\varepsilon \mathbf{1}+\mathbf{8})+\beta(\mathbf{9}),
$$

which is satisfied with

$$
\alpha=0, \beta=0 .
$$

Therefore, the subalgebra is $\{\varepsilon \mathbf{1}+\mathbf{8}, \mathbf{9}\}$. Other elements of the optimal system of the algebra $L^{9}$ are constructed in the similar way.

The list of two-dimensional subalgebras of the optimal system of the algebra $L^{9}$ is presented in Table 1.

\section{Invariant Solutions of Equation (1)}

Invariant solutions of Equation (1) are presented in this section. Analysis of invariant solutions is presented in details for two examples.

\subsection{Subalgebra 7: $\{5,4+\alpha \mathbf{9}\}$}

The basis of this subalgebra is

$$
\begin{aligned}
& X_{5}=\frac{2 t}{y} \partial_{s}-s y U \partial_{U}, \\
& X_{4}+\alpha X_{9}=2 t \partial_{t}+y \partial_{y}+(\alpha-1) U \partial_{U} .
\end{aligned}
$$

Let a function 
Table 1. Two-dimensional subalgebras of the optimal system of the algebra $L^{9}$.

\begin{tabular}{cccc}
\hline $\mathrm{N}$ & Generator & $\mathrm{N}$ & Generator \\
\hline 1 & $\mathbf{2}, \mathbf{3}$ & 11 & $\mathbf{1}, \mathbf{2}+\alpha \mathbf{4}+\mathbf{6}+\beta \mathbf{9}$ \\
2 & $\mathbf{2}, \mathbf{7}$ & 12 & $\mathbf{8}, \alpha \mathbf{4}+\mathbf{6}+\beta \mathbf{9}$ \\
3 & $\mathbf{5}, \mathbf{7}$ & 13 & $\mathbf{8}, \alpha \mathbf{4}+\mathbf{5}+\mathbf{6}+\beta \mathbf{9}$ \\
4 & $\mathbf{2}, \mathbf{3}+\varepsilon \mathbf{7}$ & 14 & $\mathbf{4}+\alpha \mathbf{9}, \mathbf{6}+\beta \mathbf{9}$ \\
5 & $\mathbf{1}, \mathbf{4}+\alpha \mathbf{9}$ & 15 & $\mathbf{6}+\alpha \mathbf{9}, \varepsilon \mathbf{1}+\mathbf{8}+\beta \mathbf{9}$ \\
6 & $\mathbf{2}, \mathbf{4}+\alpha \mathbf{9}$ & 16 & $\mathbf{2}+\mathbf{7}, \mathbf{3}+\mathbf{5}+\alpha \mathbf{7}$ \\
7 & $\mathbf{5}, \mathbf{4}+\alpha \mathbf{9}$ & 17 & $\mathbf{2}+\alpha \mathbf{9}, \mathbf{1}+\beta \mathbf{7}+\alpha \mathbf{9}$ \\
8 & $\mathbf{8}, \mathbf{4}+\alpha \mathbf{9}$ & 18 & $\mathbf{5}+\alpha \mathbf{9}, \beta \mathbf{3}+\mathbf{8}+\alpha \mathbf{9}$ \\
9 & $\mathbf{1}, \mathbf{2}+\mathbf{4}+\alpha \mathbf{9}$ & 19 & $\mathbf{2}+\varepsilon \mathbf{5}, \mathbf{- 1}+\mathbf{8}+\alpha \mathbf{9}$ \\
10 & $\mathbf{1}, \alpha \mathbf{4}+\mathbf{6}+\beta \mathbf{9}$ & 20 & $\mathbf{1}-\mathbf{8}+\mathbf{2} \alpha \mathbf{9}, \mathbf{1}+\mathbf{4}+\alpha \mathbf{9}$ \\
\hline
\end{tabular}

$$
f=f(t, s, y, U)
$$

be an invariant of the generator $X_{5}$. This means that

$$
\frac{2 t}{y} f_{s}-s y U f_{U}=0 \text {. }
$$

The general solution of this equation is

$$
f=F(t, y, \hat{U}), \hat{U}=U \mathrm{e}^{\frac{(s y)^{2}}{4 t}} .
$$

After substituting it into the equation $\left(X_{4}+\alpha X_{9}\right) f=0$, one obtains the equation

$$
2 t F_{t}+y F_{y}+(\alpha-1) \hat{U} F_{\hat{U}}=0 .
$$

The characteristic system of the last equation is

$$
\frac{\mathrm{d} t}{2 t}=\frac{\mathrm{d} y}{y}=\frac{\mathrm{d} \hat{U}}{(\alpha-1) \hat{U}} .
$$

Thus the universal invariant of this subalgebras consists of invariants

$$
\frac{y^{2}}{t}, \hat{U} y^{1-\alpha}, \hat{U}=U \mathrm{e}^{\frac{(s y)^{2}}{4 t}} .
$$

Hence, a representation of the invariant solution is

$$
U=y^{\alpha-1} \mathrm{e}^{-\frac{(s y)^{2}}{4 t}} \phi(q)
$$

with arbitrary functions $\phi(q)$ and $q=y^{2} / t$. After substituting this representation into Equation (1), one obtains the ordinary differential equation

$$
8 q^{2} \phi^{\prime \prime}+2 q(q+4 \alpha-2) \phi^{\prime}+\left(2 \alpha^{2}-6 \alpha-q+4\right) \phi=0 .
$$

The general solution of the last equation is

$$
=\mathrm{e}^{-\frac{q}{8}} q^{\frac{2 \alpha-3}{4}}\left[C_{1} W_{1}\left(\frac{2 \alpha-1}{4}, \frac{1}{4}, \frac{q}{4}\right)+C_{2} W_{2}\left(\frac{2 \alpha-1}{4}, \frac{1}{4}, \frac{q}{4}\right)\right],
$$

where $W_{1}\left(\frac{2 \alpha-1}{4}, \frac{1}{4}, \frac{q}{4}\right), W_{2}\left(\frac{2 \alpha-1}{4}, \frac{1}{4}, \frac{q}{4}\right)$ are Whittaker functions and $C_{1}, C_{2}$ are arbitrary constants.

\subsection{Subalgebra 16: $\{2+7,3+5+\alpha 7\}$}

The basis of this subalgebra consists of the generators

$$
\begin{aligned}
& X_{2}+X_{7}=\left(\frac{1+2 t s}{y}\right) \partial_{s}-2 t \partial_{y}+y U \partial_{U}, \\
& X_{3}+X_{5}+\alpha X_{7}=\left(\frac{s+(1+\alpha s) 2 t}{y}\right) \partial_{s} \\
& -(1+2 \alpha t) \partial_{y}+(\alpha-s) y U \partial_{U} .
\end{aligned}
$$

In order to find an invariant solution, one needs to find a universal invariant of this subalgebra. Let a function

$$
f=f(t, s, y, U)
$$

be an invariant of the generator $X_{2}+X_{7}$. This means that

$$
\left(\frac{1+2 t s}{y}\right) f_{s}-2 t f_{y}+y U f_{U}=0
$$

The characteristic system of the last equation is

$$
\frac{y \mathrm{~d} s}{(1+2 t s)}=\frac{\mathrm{d} y}{-2 t}=\frac{\mathrm{d} U}{y U}=\frac{\mathrm{d} t}{0} .
$$

The general solution of this equation is

$$
f=F(t, \hat{y}, \hat{U}), \hat{y}=y(2 t s+1), \hat{U}=U \mathrm{e}^{\frac{y^{2}}{4 t}} \text {. }
$$

After substituting it into the equation

$$
\left(X_{3}+X_{5}+\alpha X_{7}\right) f=0
$$

one obtains the equation

$$
2 t\left(1+2 \alpha t-4 t^{2}\right) F_{\hat{y}}+\hat{y} \hat{U} F_{\hat{U}}=0 .
$$

The characteristic system of this equation is

$$
\frac{\mathrm{d} \hat{y}}{2 t\left(1+2 \alpha t-4 t^{2}\right)}=\frac{\mathrm{d} \hat{U}}{\hat{y} \hat{U}}=\frac{\mathrm{d} t}{0} .
$$

Hence, the universal invariant of this subalgebras consists of invariants

$$
t, \hat{U} \mathrm{e}^{-\frac{\hat{y}^{2}}{4 t\left(1+2 \alpha t-4 t^{2}\right)}}, \hat{y}=y(2 t s+1), \hat{U}=U \mathrm{e}^{\frac{y^{2}}{4 t}} .
$$

A representation of the invariant solution of this subalgebra has the following form

$$
U=\mathrm{e}^{\frac{(y(2 t s+1))^{2}}{4 t\left(1+2 \alpha t-4 t^{2}\right)}-\frac{y^{2}}{4 t}} \phi(t)
$$

with an arbitrary function $\phi(t)$. After substituting the 
representation of the invariant solution into Equation (1), the functions $\phi(t)$ has to satisfy the equation

$$
\left(1+2 \alpha t-4 t^{2}\right) \phi^{\prime}+(\alpha-4 t) \phi=0 .
$$

The general solution of the last equation is

$$
\phi=C / \sqrt{1+2 \alpha t-4 t^{2}}
$$

where $C$ is constant.

The two examples showed that there are solutions of the Navier-Stokes equations, which are partially invariant with respect to not admitted Lie algebra $t \partial_{t}+x \partial_{x}+y \partial_{y}+z \partial_{z}$.

\section{Conclusion}

The algorithm of obtaining an optimal system of subalgebras was applied to the reduction of the NavierStokes equations. Some exact invariant solutions corresponding to the optimal system are presented. Examples given in the manuscript showed that this algorithm can be applied to groups, which are not admitted. These possibilities extend an area of using group analysis for constructing exact solutions.

\section{Acknowledgements}

This research is supported by the Centre of Excellence in Mathematics, the Commission on Higher Education, Thailand.

\section{REFERENCES}

[1] L. V. Ovsiannikov and A. P. Chupakhin, "Regular Partially Invariant Submodels of the Equations of Gas Dynamics," Journal of Applied Mechanics and Technics, Vol. 6, No. 60, 1996, pp. 990-999.

[2] S. Lie, "On General Theory of Partial Differential Equations of an Arbitrary Order," German, No. 4, 1895, pp. 320-384.

[3] L. V. Ovsiannikov, "Partly Invariant Solutions of the Equations Admitting a Group," Proceedings of the 11th International Congress of Applied Mechanics, SpringerVerlag, Berlin, 1964, pp. 868-870.

[4] V. V. Pukhnachov, "Group Properties of the NavierStokes Equations in Two-Dimensional Case," Journal of Applied Mechanics and Technical Physics, Vol. 44, No. 3, 1960, pp. 317-323. doi:10.1023/A:1023472921305

[5] V. O. Bytev, "Group Properties of Navier-Stokes Equations," Chislennye Metody Mehaniki Sploshnoi Sredy, Vol. 3, No. 3, 1972, pp. 13-17.

[6] S. V. Khabirov, "Partially Invariant Solutions of Equations of Hydrodynamics," Exact Solutions of Differential Equations and Their Assymptotics, Ufa, 1992.

[7] B. J. Cantwell, "Introduction to Symmetry Analysis," Camridge University Press, Camridge, 2002.

[8] B. J. Cantwell, "Similarity Transformations for the Two-
Dimensional, Unsteady, Stream-Function Equation," Journal of Fluid Mechanics, Vol. 85, No. 2, 1978, pp. 257271. doi:10.1017/S0022112078000634

[9] S. P. Lloyd, "The Infinitesimal Group of the NavierStokes Equations," Acta Mathematica, Vol. 38, 1981, pp. 85-98.

[10] R. E. Boisvert, W. F. Ames and U. N. Srivastava, "Group Properties and New Solutions of Navier-Stokes Equations," Journal of Engineering Mathematics, Vol. 17, 1983, pp. 203-221. doi:10.1007/BF00036717

[11] A. Grauel and W. H. Steeb, "Similarity Solutions of the Euler Equation and the Navier-Stokes Equations in Two Space Dimensions," International Journal of Theoretical Physics, No. 24, No. 3, 1985, pp. 255-265. doi:10.1007/BF00669790

[12] N. H. Ibragimov and G. Unal, "Equivalence Transformations of Navier-Stokes Equation," Bulletin of the Technical University of Istanbul, Vol. 1-2, No. 47, 1994, pp. 203-207.

[13] R. O. Popovych, "On Lie Reduction of the Navier-Stokes Equations," Nonlinear Mathematical Physics, Vol. 3-4, No. 2, 1995, pp. 301-311. doi:10.2991/jnmp.1995.2.3-4.10

[14] W. I. Fushchich and R. O. Popovych, "Symmetry Reduction and Exact Solution of the Navier-Stokes Equations," Nonlinear Mathematical Physics, Vol. 1, No. 1, 1994, pp. 75-113. doi:10.2991/jnmp.1994.1.1.6

[15] W. I. Fushchich and R. O. Popovych, "Symmetry Reduction and Exact Solution of the Navier-Stokes Equations," Nonlinear Mathematical Physics, Vol. 2, No. 1, 1994, pp. 158-188. doi:10.2991/jnmp.1994.1.2.3

[16] D. K. Ludlow, P. A. Clarkson and A. P. Bassom, "Similarity Reduction and Exact Solutions for the Two-Dimensional Incompressible Navier-Stokes Equations," Studies in Applied Mathematics, Vol. 103, 1999, pp. 183240. doi:10.1111/1467-9590.00125

[17] V. V. Pukhnachov, "Free Boundary Problems of the Navier-Stokes Equations," Doctoral Thesis, Novosibirsk, 1974.

[18] A. F. Sidorov, V. P. Shapeev and N. N. Yanenko, "The Method of Differential Constraints and Its Applications in Gas Dynamics," Nauka, Novosibirsk, 1984.

[19] S. V. Meleshko, "Classification of the Solutions with Degenerate Hodograph of the Gas Dynamics and Plasticity Equations," Doctoral Thesis, Ekaterinburg, 1991.

[20] S. V. Meleshko, "One Class of Partial Invariant Solutions of Plane Gas Flows," Differential Equations, Vol. 10, No. 30, 1994, pp. 1690-1693.

[21] L. V. Ovsiannikov, "Isobaric Motions of a Gas," Differential Equations, Vol. 10, No. 30, 1994, pp. 1792-1799.

[22] A. P. Chupakhin, "On Barochronic Motions of a Gas," Doklady Rossijskoj Akademii Nauk, Vol. 5, No. 352, 1997, pp. 624-626.

[23] A. M. Grundland and L. Lalague, "Invariant and partially Invariant Solutions of the Equations Describing A NonStationary and Isotropic Flow for an Ideal and Compressible Fluid in (3+1) Dimensions," Journal of Physics 
A: Mathematical and General, Vol. 29, No. 8, 1996, pp. 1723-1739. doi:10.1088/0305-4470/29/8/019

[24] L. V. Ovsiannikov, "Group Analysis of Differential Equations," Nauka, Moscow, 1978.

[25] L. V. Ovsiannikov, "Regular and Irregular Partially In- variant Solutions," Doklady Academy of Sciences of USSR, Vol. 2, No. 343, 1995, pp. 156-159.

[26] L. V. Ovsiannikov, "Program SUBMODELS. Gas Dynamics," Journal of Applied Mathematics and Mechanics, Vol. 58, 1994, pp. 30-55. 